\title{
Heparin Induced Extracorporeal LDL Precipitation (H.E.L.P.) in Treat- ment of Tinnitus: A Randomised, Multicentre Trial
}

\author{
M. Canis ${ }^{1}$, F. Blessing ${ }^{2}$, B. Mazurek ${ }^{3}$, U. Kassner ${ }^{4}$, A. Vogt ${ }^{4}$, D. Osterkorn ${ }^{5}$, K. Osterkorn ${ }^{5}$, \\ M. Suckfuell ${ }^{*}, 1$ \\ ${ }^{I}$ Department of Otorhinolaryngology, Head and Neck Surgery, University of Munich, Germany \\ ${ }^{2}$ Institute for Clinical Chemistry, University of Munich, Germany \\ ${ }^{3}$ Tinnitus Center, Department of Otorhinolaryngology, Head and Neck Surgery, University of Berlin, Germany \\ ${ }^{4}$ Lipidclinic, Department of Medicine, Charité Universitätsmedizin Berlin, Germany \\ ${ }^{5}$ Medizinisches Wirtschaftsinstitut, Munich, Germany
}

\begin{abstract}
Background: At the beginning, cochlear tinnitus seems to arise from a dysfunction of cochlear outer hair cells. Similar to other inner ear disorders a disturbance of cochlear microcirculation is one of the most frequently discussed reasons. Animal studies and clinical evidence show a negative effect of high cholesterol and fibrinogen on hearing function. Acutely lowering serum LDL and fibrinogen by means of LDL and fibrinogen apheresis increases cochlear blood flow and has been proven to be effective in treatment of idiopathic sudden sensorineural hearing loss. Therefore we aimed to determine whether acute reduction of plasma fibrinogen and serum LDL is effective for treatment of subacute tinnitus.

Methods: Between January, 2004 and December, 2006 we included patients of 18 through 80 years of age suffering from subacute cochlear tinnitus between 3 to 12 months on one or both ears and having serum LDL-cholesterol between 130 and $190 \mathrm{mg} / \mathrm{dl}$. Tinnitus was evaluated using a standardised questionnaire designed by Goebel and Hiller. Patients meeting inclusion criteria were provided with the HMG-CoA reductase inhibitor simvastatin $40 \mathrm{mg}$ once daily for 3 months. This procedure was done due to ethical reasons to reduce cholesterol and LDL non invasively in a first step. After 3 months tinnitus was re-evaluated and patients were excluded from the study if severity fell by more than 20 on the tinnitus score. 27 included participants were randomly assigned in a one to one ratio either to triple fibrinogen/LDL apheresis or triple sham therapy. Differences between tinnitus score on the day of randomisation and after 12 weeks were used as main outcome measure. Development of tinnitus score after every treatment, differences in loudness and frequency of tinnitus (masking and matching) after every treatment and after 12 weeks and the development of hearing thresholds after 12 weeks were used as secondary outcome measures.

Findings: Main outcome measure tinnitus score 12 weeks after first therapy showed no significant differences between the groups. Though, there was a strong tendency towards an relieve of tinnitus in the apheresis group $(p=0.069$ for the difference of the baseline value and the 12 weeks value between apheresis $v s$ sham-therapy). Neither pure tone thresholds after 12 weeks, nor tinnitus masking and matching after every therapy and after 12 weeks showed significant differences between the groups.

Conclusions: In our study, all patients suffered from subacute tinnitus and tinnitus score was stable or slightly worse in the placebo group over time. The improvement of tinnitus score in the apheresis group - although not statistically significant - is therefore a remarkable observation. For a more conclusive evaluation of this observation further studies with a larger number of patients should be conducted.
\end{abstract}

\section{INTRODUCTION}

At the beginning, cochlear tinnitus seems to arise from a dysfunction of cochlear outer hair cells. However, the underlying pathomechanisms are still obscure. Similar to other inner ear disorders like sudden sensorineural hearing loss, disturbance of cochlear microcirculation is one of the most frequently discussed reasons. Cochlear blood flow is sensitive to changes and even limited impairment of perfusion leads to an immediate dysfunction of the organ of corti [1]. Animal studies and clinical evidence show a negative effect

*Address correspondence to this author at the Ludwig-MaximiliansUniversität München, Klinikum Großhadern, Marchioninistr. 15, D-81377 München, Germany; E-mail: markus.suckfuell@med.uni-muenchen.de of hyperlipidemia on hearing function [2]. High serum LDL (low density lipoprotein) and low HDL (high density lipoprotein) are commonly accepted as vascular risk factors. Beyond from its well known part in development of atherosclerosis and increase of blood viscosity, cholesterol can impair cochlear microcirculation by diminishing the release of the potent vasodilator nitric oxide (NO) from endothelial cells [3]. A second possible mechanism of hearing impairment by high serum cholesterol is a direct action at the outer hair cell $(\mathrm{OHC})$ membrane. Isolated $\mathrm{OHCs}$ show diminished motility when incubated with a cholesterol enriched medium, probably due to increased stiffness caused by integration of cholesterol molecules into the lateral wall membrane [4]. Since fibrinogen is a large glycoprotein that defines rheological properties of whole blood by increasing plasma 
viscosity and inducing aggregation of erythrocytes, thrombocytes and leucoytes it is also thought to be a risk factor for inner ear disorders by reducing cochlear blood flow $[5,6]$. Acutely lowering serum LDL and fibrinogen by means of LDL and fibrinogen apheresis thus increases cochlear blood flow without additional haemodilution and has been proven to be effective in treatment of idiopathic sudden sensorineural hearing loss. Interestingly, a minor $(1,8 \mathrm{~dB})$ but significant improvement of hearing threshold at the contralateral, healthy ear was also observed in the study [7]. Within the last years apheresis was used as therapeutic option in treatment of sudden sensorineural hearing loss in particular if standard treatment with steroids or hemodilution were ineffective. In clinical experience many of these patients do not only report an improvement of hearing threshold but also a reduction of tinnitus. We therefore were confronted with the question if patients with continuing tinnitus also benefit from apheresis treatment. As tinnitus is a disease with a high emotional contribution sham treatment and the use of a validated questionnaire assessing the impact of tinnitus on the individual were necessary to answer this question. Because of the relevance for these patients we aimed to assess whether lowering serum fibrinogen and LDL levels by fibrinogen/LDL apheresis may also be an effective treatment of tinnitus.

\section{METHODS}

\section{Study Design}

A prospective, randomized, single-blind clinical trial meeting GCP criteria was performed.

\section{Settings and Locations}

Patients were recruited from the ENT departments of the University of Munich and the University of Berlin between January, 2004 and December, 2006. The study was done in accordance with the declaration of Helsinki and the study protocol was approved by the local ethics committees. All patients provided written informed consent. An independent office (Medizinisches Wirtschaftsinstitut, Munich, Germany) monitored and analysed the data.

\section{Participants}

We included patients of 18 through 80 years of age suffering from subacute cochlear tinnitus between 3 to 9 months duration on one or both ears and having serum LDLcholesterol between 130 and $190 \mathrm{mg} / \mathrm{dl}$. Tinnitus was evaluated using a standardised questionnaire designed by Goebel and Hiller [8]. Patients were eligible if tinnitus score was 30 to 60 (moderate to severe intensity). Audiometric testing included pure-tone audiometry (frequencies 125, 250, 500, $1000,2000,3000,4000,6000$ and $8000 \mathrm{~Hz}$ ) in accordance with ISO 7029, tympanometry, stapedius reflex measurements and the German speech intelligibility (Freiburger Sprachtest). The sound level in $\mathrm{dB}$ at which $50 \%$ of the recorded digits were recognised corresponds to perception of speech. Furthermore masking and matching of the tinnitus were performed. Laboratory tests were done at the departments of clinical chemistry of the two participating university clinics with standard methods including total cholesterol, HDL cholesterol, LDL cholesterol, triglycerides and lipoprotein (a).

Patients were excluded if they had previously been treated for tinnitus, had objective or retrocochlear tinnitus, other disorders of the inner ear with known cause, Menière's disease, subacute, conductive or psychogenic hearing loss. We also excluded patients if they had a clotting disorder, malignant disease, heart failure (NYHA III or IV), arrythmia, haemodialysis, hapatitis B or C, HIV-1, HIV-2 or dementia.

\section{Interventions}

Before enrolement patients meeting inclusion criteria were provided with the HMG-CoA reductase inhibitor simvastatin $40 \mathrm{mg}$ once daily for 3 months. This procedure was suggested by the ethic committees to reduce cholesterol and LDL non invasively in a first step. After 3 months tinnitus was re-evaluated and patients were excluded from the study if severity fell by more than 20 on the tinnitus score describing at least one step into the next severtity group. Eligible patients were enrolled into the study and gave written informed consent. Included participants then were randomly assigned in a one to one ratio either to triple fibrinogen/LDL apheresis (B Braun, Melsungen, Germany) or triple sham therapy. Centres were provided with sealed cards containing randomisation codes to assign the patients to one of the two treatments. The randomisation code was developed with computerised random number generator. An independent medical doctor from the ENT clinic obtained informed consent from the patients, enrolled them and ascertained the individual treatment. Because of the substantial difference of the two treatment groups only patients but not the investigators were blinded during therapy.

The procedure of fibrinogen/LDL apheresis has been described previously. Briefly, plasma is obtained by filtration of whole blood through a $0.5 \mu \mathrm{m}$ filter and then mixed continuously with an equal volume of a $0.3 \mathrm{M}$ sodium acetate buffer (pH 4.85) containing $100 \mathrm{IU} / \mathrm{ml}$ heparin. The solution precipitates at a final $\mathrm{pH}$ of around 5.12. The suspension is continuously recirculated through a $0.45 \mu \mathrm{m}$ polyethersulfone filter from which plasma free from LDL cholesterol and fibrinogen is obtained and then passed through an anion exchange filter to adsorb excess heparin. Finally, we restored the physiological $\mathrm{pH}$ by bicarbonate dialysis and removed excess fluid by ultrafiltration before mixing the plasma with the blood cells and returning the solution to the patient. We treated 31 plasma within 2 hours with a machine that monitors and controls apheresis (Plasmat ${ }^{\mathbb{B}}$ Futura, B Braun Medizintechnologie GmbH, Melsungen, Germany) on an outpatient basis. This procedure was done once a week in 3 consecutive weeks and reduced plasma cholesterol, LDL, fibrinogen and lipoprotein (a) by more than $50 \%$. After vein puncture patients were blinded during the whole procedure by wearing eye mask and ear muffs.

For sham therapy patients were blinded after vein puncture and connected to the apheresis machine running in idle mode without any blood flow for about $2 \mathrm{~h}$ (also see Fig. (1)).

Audiologic testing was performed as described above before every treatment, 3, 6 and 12 weeks after the start of therapy. Laboratory tests were done before and after every treatment, 3, 6 and 12 weeks after the start of therapy. We measured the concentrations of sodium, potassium, calcium, urea, glucose, creatinine, total protein, bilirubin, uric acid, albumin, total cholesterol, HDL cholesterol, LDL cholesterol, triglyceride, lipoprotein (a), high sensitive C-reactive protein, alanine 


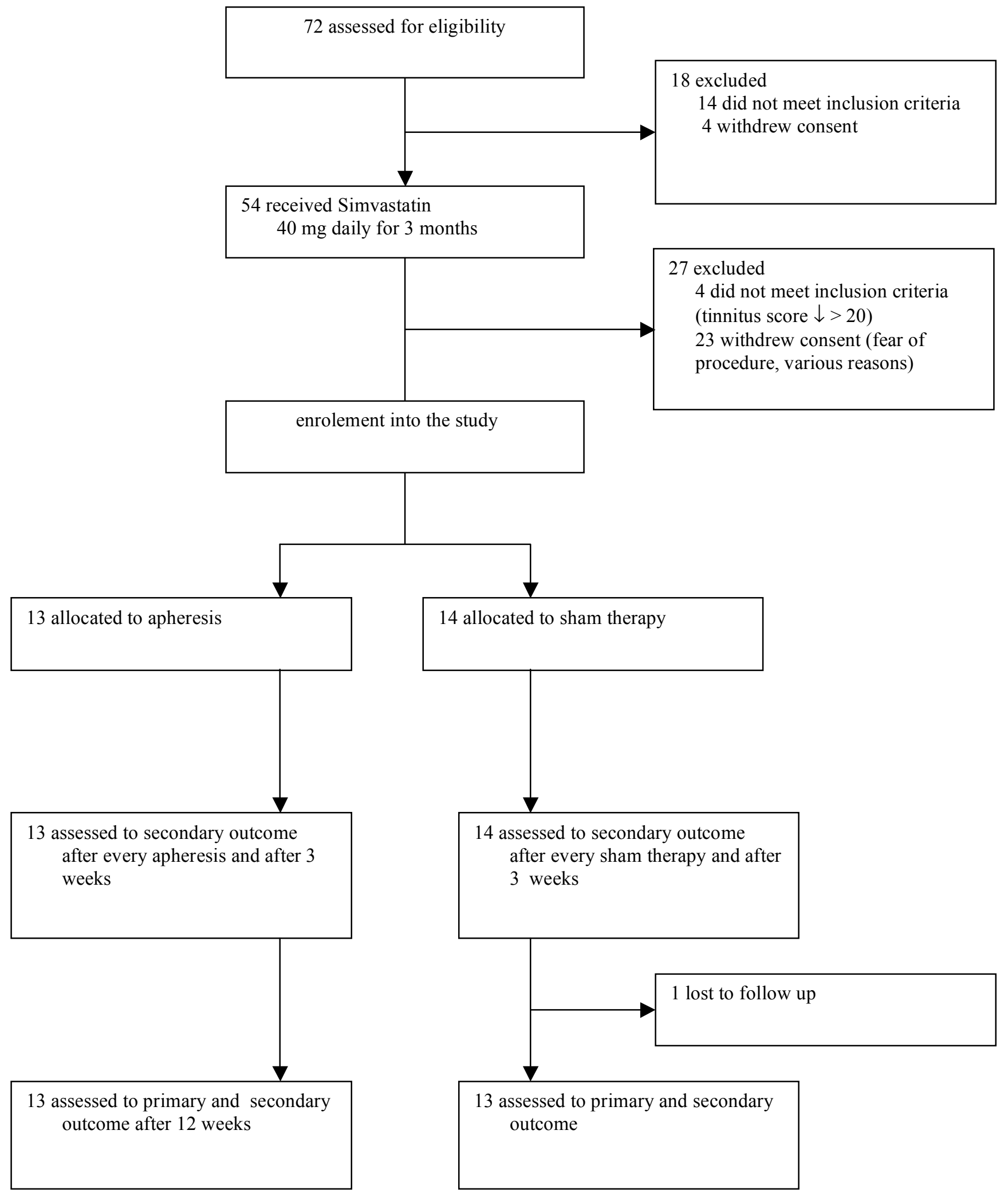

Fig. (1). Trial profile.

transaminase and aspartate tranaminase. We also assessed leucocytes, erythrocytes, thrombocytes, haemoglobin, packedcell volume, mean corpuscular volume, mean corpuscular haemoglobin, mean corpuscular haemoglobin concentration, the concentration of fibrinogen, the international normalisation ratio and the partial thrombine time.

\section{Outcome Measures and Statistical Analysis}

We aimed to test the hypothesis that triple fibrinogen/LDL apheresis is more effective in treatment of tinnitus than triple sham therapy. Effectiveness was assumed when main outcome measure was significantly better in the 


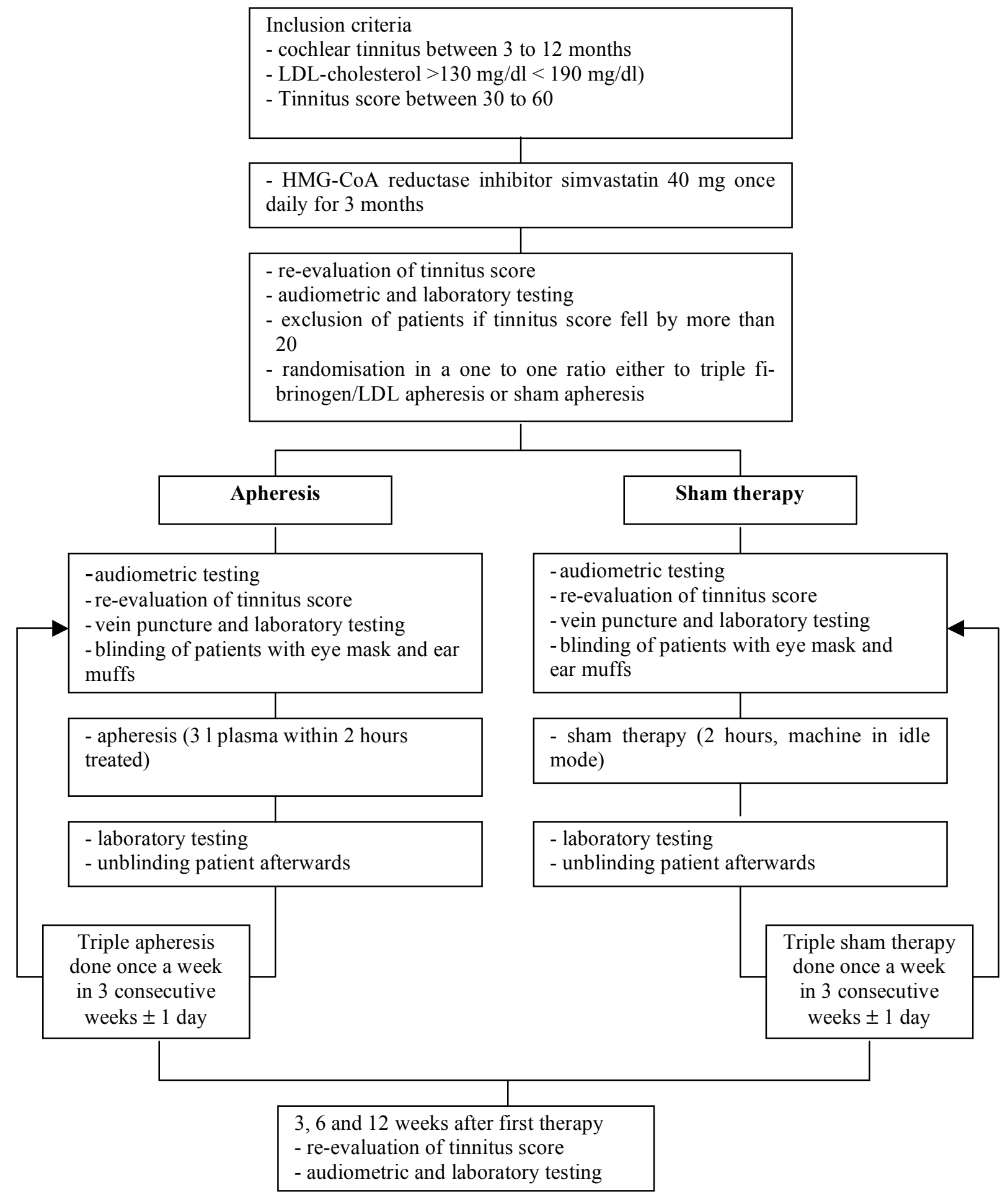

Fig. (2). Treatment profile.

apheresis group than in the sham therapy group. Differences between tinnitus score on the day of randomisation and after 12 weeks were used as main outcome measure. Development of tinnitus score after every treatment, differences in loudness and frequency of tinnitus (masking and matching) after every treatment and after 12 weeks and the development of hearing thresholds (pure tone audiometry at frequencies of 3 , 4,6 and $8 \mathrm{kHz}$ ) after 12 weeks were used as secondary outcome measures. Analysis of data was restricted to those par- ticipants who adhered fully to the protocol in relation to eligibility, interventions and main outcome assessment (per protocol analysis). The descriptive and confirmatory analyses were done with the SAS System (Version 9.1) and SPSS System (Version 13.0) for Windows. The Wilcoxon signed rank-test was used for comparison between the two groups. A p-value of $\alpha \leq 0.05$ was judged significant. 


\section{RESULTS}

54 patients met inclusion criteria and were provided with Simvastatin $40 \mathrm{mg}$ once daily. After 3 month 4 patients were excluded because tinnitus score fell by more than 20 . However, further analysis and comparison of these patients showed no concomitant factors in its baseline characteristics. 23 patients withdrew their consent after therapy with Simvastatin but before randomisation for various reasons. Mainly the fear of the invasive procedure was the intention to withdraw. We randomly allocated 13 patients to the apheresis group and 14 to the sham therapy group (Fig. 2). Table 1 shows the participants' baseline characteristics. Sex distribution, age, body-mass index and blood pressure did not differ significantly between the groups. We were able to assess the primary outcome in 13 participants in the apheresis group and in 13 patients in the sham therapy group. 1 patient was lost to follow up 12 weeks after first sham therapy. We could assess secondary outcome measures in 13 patients in the apheresis group and in 14 patients in the sham therapy group until 3 weeks after first therapy. Secondary outcome measures 12 weeks after randomisation were measured in 13 participants in the sham therapy group. Fig. (1) shows the trial profile. Table 2 shows the laboratory data before and after every therapy and 12 weeks after first treatment. Patients receiving apheresis had significant $(\mathrm{p}<0,01)$, substantial reductions of total cholesterol, LDL cholesterol, triglycerides, lipoprotein (a) and fibrinogen whereas in the sham therapy group no significant reduction was seen. 1 week after every apheresis all patients returned back to their baseline values of the blood chemical tests done.

\section{Table 1. Patient's Baseline Characteristics}

\begin{tabular}{|c|c|c|}
\hline & $\begin{array}{c}\text { Apheresis } \\
(n=13)\end{array}$ & $\begin{array}{l}\text { Sham } \\
(n=14)\end{array}$ \\
\hline \multicolumn{3}{|l|}{ Characteristics } \\
\hline Sex & & \\
\hline Male & $\mathrm{n}=9$ & $\mathrm{n}=10$ \\
\hline Female & $\mathrm{n}=4$ & $\mathrm{n}=4$ \\
\hline Age & $55.4 \pm 11.8$ & $52.8 \pm 8.5$ \\
\hline Mean duration of tinnitus (months) & $7 \pm 4$ & $8 \pm 3$ \\
\hline Body-mass index $\left(\mathrm{kg} / \mathrm{m}^{2}\right)$ & $26.7 \pm 4.0$ & $28.6 \pm 4.0$ \\
\hline Blood pressure (mm Hg) & & \\
\hline systolic & $136.0 \pm 20.0$ & $142.2 \pm 18.9$ \\
\hline diastolic & $85.5 \pm 13.0$ & $86.7 \pm 6.6$ \\
\hline Heart rate (beats/min) & $71.7 \pm 10.8$ & $81.0 \pm 7.6$ \\
\hline Smokers & $5(38 \%)$ & $9(65 \%)$ \\
\hline
\end{tabular}

Fig. (3) shows the development of tinnitus score. Main outcome measure tinnitus score 12 weeks after first therapy showed no significant differences between the groups. Though, there was a tendency towards a relieve of tinnitus in the apheresis group. Total tinnitus score fell from 36.9 to 36.5 after 1, to 34.2 after 2 , to 33.8 after 3 and to 30.5 after 12 weeks in the apheresis group while it rose in the placebo group from 43.1 to 46.7 after 1, to 48.3 after 2, to 48.7 after
3 and to 44.7 after 12 weeks ( $p=0.069$ for the difference of the baseline value and the 12 weeks value between apheresis $v s$ sham-therapy). Neither pure tone thresholds after 12 weeks, nor tinnitus masking and matching after every therapy and after 12 weeks showed significant differences between the groups.

\section{DISCUSSION}

Since $80 \%$ of patients suffering from sudden sensorineural hearing loss also complain about tinnitus [5] a dysfunction of outer hair cells at least in the acute and subacute state may be the main cause. In many cases hearing loss is not critical enough to be directly experienced by the patients but nevertheless may result in tinnitus of the affected frequency. However, until today little is known about the underlying pathomechanisms. Experimental and clinical data suggest that elevated serum cholesterol and fibrinogen might be cofactors in the development of hearing disorders [3,4,7,9-11]. Fibrinogen/LDL apheresis is an established procedure to acutely reduce the concentration of serum LDL and lipoprotein (a) to $60 \%$ and that of fibrinogen to $65 \%$ of the original values. Reduction of plasma fibrinogen improves the blood's rheological properties and results in a significant reduction of plasma viscosity and an increase in tissue oxygenation. This effect is well known and can be measured in muscle for example by polarographic needle methods $[12,13]$. Furthermore, a very large and quick reduction of plasma LDL cholesterol by apheresis improves endothelial functions in coronary and peripheral arteries [14] as shown by positron emission tomography [15]. This substantial improvement of haemorheology and endothelial function thus also might become relevant in cochlear circulation. High serum LDL and low HDL are commonly accepted as major vascular risk factors. Many authors assume that hyperlipidemia with the subsequent hyperviscosity of the blood and atherosclerosis reduce the cochlear perfusion and thus may trigger disorders in hearing function $[9,16,17]$. Indeed, histochemical studies on the inner ear of hypercholesterolemic animals showed a vacuolar degeneration of the capillary vessels of the stria vascularis [18] and elctro-licent patches of an amorphous material in strial marginal cells throughout the cochlea and in $\mathrm{OHC}$ [19]. The authors hypothesize that these changes indicate metabolic stress and may result in cochlear dysfunction. In adidition to the vascular and haemorheological effects of apheresis, the direct effect on plasma cholesterol concentrations by such a treatment could also affect the perilymph compartment and the composition of outer hair cell membranes in particular the ratio of phospholipids to cholesterol. Nguyen and Brownell [4] showed in vitro that the lateral wall of OHCs from the guinea pig cochlea can incorporate water-soluble cholesterol and thus may interact with the OHC's membrane directly. This uptake of cholesterol is accompanied by an increased stiffness of the cells. They suggest that increased cholesterol-induced cell stiffness may impair the electromotile response of OHCs and thus disturb inner ear function because fast motility of $\mathrm{OHCs}$ is the physiologic basis for cochlear amplification of low sound intensities. Another pathomechanism has been proposed by Feron et al. [3]. The uptake and retention of cholesterol in endothelial cells causes diminished release of nitric oxide (NO) from endothelial cells. NO is a potent vasodilator that 
Table 2. Laboratory Data

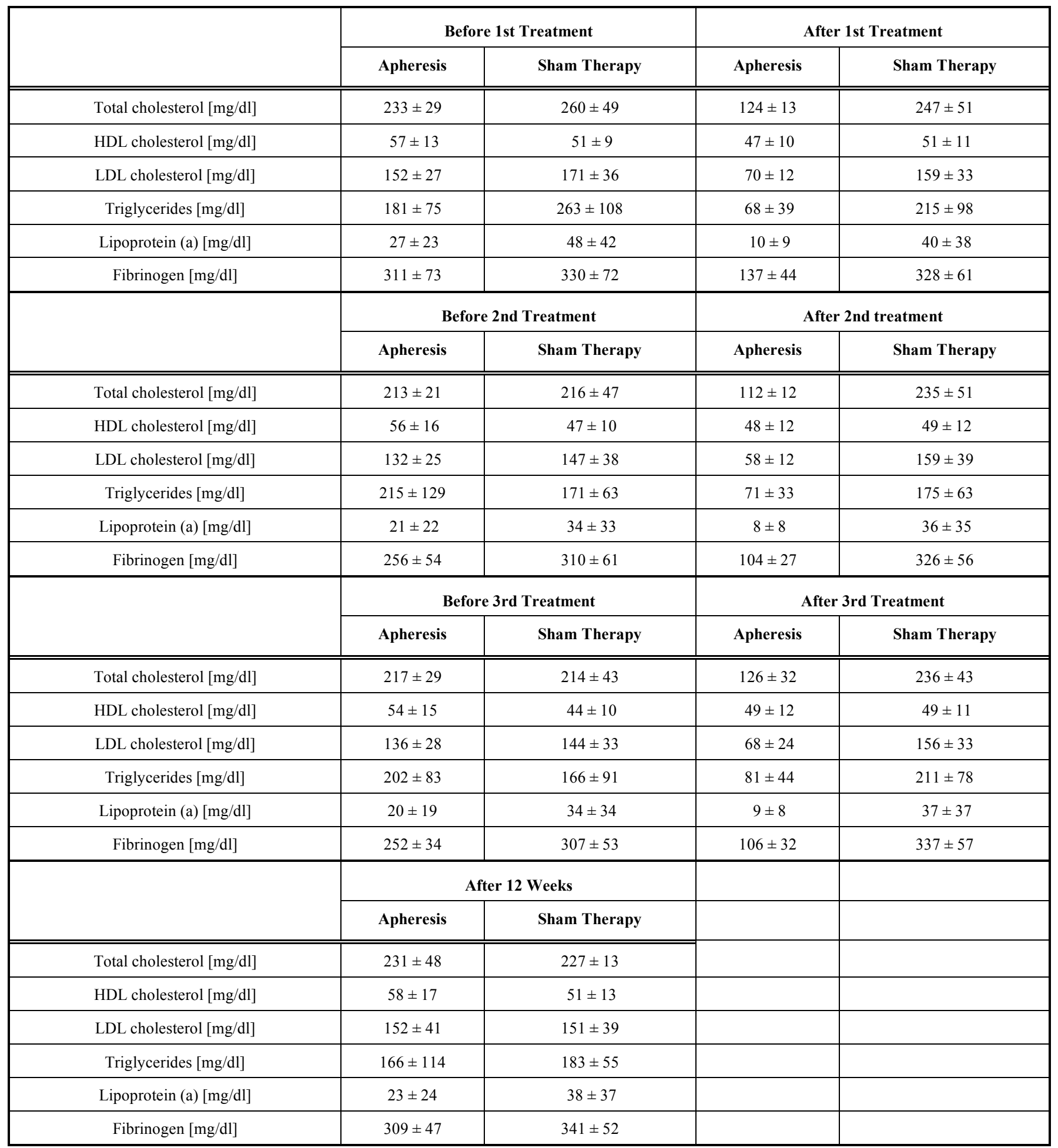

has been shown to play a crucial part in regulation of cochlear perfusion [20]. High serum cholesterol can thus impair cochlear microcirculation and contribute to consecutive cochlear damage. Treatment to lower fibrinogen increases cochlear blood flow in animals and has been used in patients with sudden sensorineural hearing loss. In a prospective study Shiraishi et al. showed [21] a better hearing recovery in patients by lowering fibrinogen due to the venom enzyme batroxobin in comparison to steroids. Acutely lowering fi- brinogen and serum LDL by means of LDL and fibrinogen apheresis has also been proven effective in a clinical trial. In a prospective randomized clinical study Suckfuell et al. [7] showed that pure-tone thresholds in patients with sudden hearing loss improved more, but not significantly due to apheresis compared to plasmaexpander and prednisolone treatment. Interestingly, also a minor but significant elevation of the hearing threshold of the healthy contralateral ear was observed in this study. This improvement might not be 
recognized by the patients in regard of hearing but nevertheless may lead to a reduction of tinnitus. Therefore, with respect to pathophysiological hints and clinical evidence that high serum cholesterol and fibrinogen might be factors in the pathogenesis of tinnitus we aimed to assess whether lowering serum fibrinogen and LDL levels by fibrinogen/LDL apheresis may be an effective treatment of subacute tinnitus. Subacute tinnitus was chosen assuming a low rate of spontaneous improvement compared to patients suffering from acute tinnitus but still having the possibility to treat a dysfunction of the cochlea in a causal way whereas in chronic tinnitus a central nervous component gets more relevant. Our results show that tinnitus score 12 weeks after first therapy improved more but not significantly $(p=0.069)$ with apheresis treatment compared to sham-therapy. In cases of acute tinnitus, several treatment options exist. In many cases acute tinnitus even disappears without any treatment. In cases of subacute tinnitus, though, spontaneous improvement is rare and to date no causal treatment exists. The only way to help patients with chronic tinnitus is helping them to cope with the ringing in their ears by means of psychological strategies. In our study, all patients suffered from subacute tinnitus and tinnitus score was stable or slightly worse in the placebo group. The improvement of tinnitus score in the apheresis group - although not statistically significant - is therefore a remarkable observation. For a more conclusive answer whether there could be a position of this therapeutic approach in the treatment of tinnitus further studies with a larger number of patients should be conducted. However, the experience of this study may be important for the potential design of this future clinical trails.

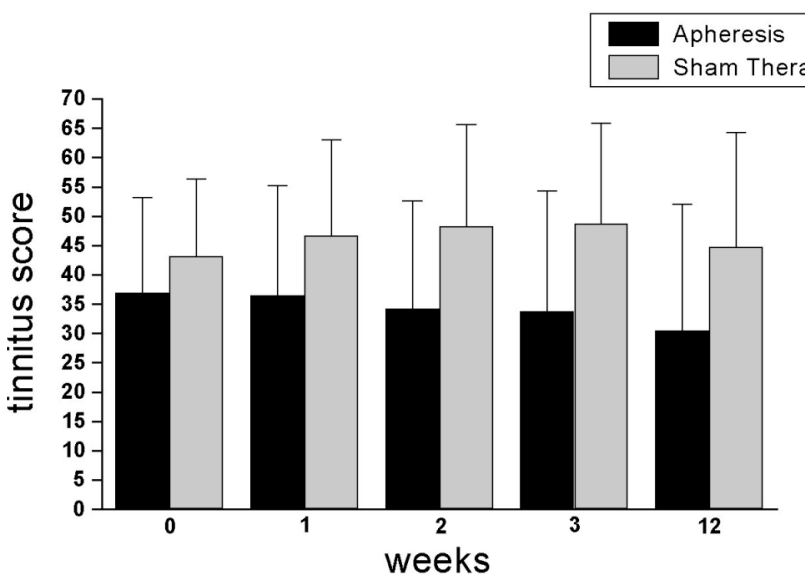

Fig. (3). Development of tinnitus score before and 12 weeks after first treatment.

\section{REFERENCES}

[1] Miller JM, Ren TY, Nuttall AL. Studies of inner ear blood flow in animals and human beings. Otolaryngol Head Neck Surg 1995; 112: 101-113.

[2] Sidman JD, Prazma J, Pulver SH, et al. Cochlea and heart as endorgans in small vessel disease. Ann Otol Rhinol Laryngol 1988; 97 : 9-13.

[3] Feron O, Dessy C, Desager JP, et al. Hydroxy-methylglutarylcoenzyme A reductase inhibition promotes endothelial nitric oxide synthase activation through a decrease in caveolin abundance. Circulation 2001; 103: 113-118.

[4] Nguyen TV, Brownell WE. Contribution of membrane cholesterol to outer hair cell lateral wall stiffness. Otolaryngol Head Neck Surg 1998; 119: 14-20.

[5] Suckfull M, Seidel D, Thiery J, et al. Treatment of sudden hearing loss through Fibrinogen/LDL-apheresis. A prospective, randomized multicenter trial. Z Kardiol 2003; 92: III59-III63.

[6] Suzuki K, Kaneko M, Murai K. Influence of serum lipids on auditory function. Laryngoscope 2000; 110: 1736-1738.

[7] Suckfull M. Fibrinogen and LDL apheresis in treatment of sudden hearing loss: a randomised multicentre trial. Lancet 2002; 360: 1811-1817.

[8] Goebel G, Hiller W. The tinnitus questionnaire. A standard instrument for grading the degree of tinnitus. Results of a multicenter study with the tinnitus questionnaire. HNO 1994; 42: 166-172.

[9] Axelsson A, Lindgren F. Is there a relationship between hypercholesterolaemia and noise-induced hearing loss? Acta Otolaryngol 1985; 100: 379-386.

[10] Preyer S, Baisch A, Bless D, et al. Distortion product otoacoustic emissions in human hypercholesterolemia. Hear Res 2001; 152: 139-151.

[11] Rosen S, Bergman M, Plster D, et al. Presbycusis study of a relatively noise-free population in the Sudan. Trans Am Otol Soc 1962; 50: $135-152$.

[12] Kojima Y, Ito S, Furuya N. Hearing improvement after therapy for hyperlipidemia in patients with chronic-phase sudden deafness. Ann Otol Rhinol Laryngol 2001; 110: 105-108.

[13] Seidel D. H.E.L.P. apheresis therapy in the treatment of severe hypercholesterolemia: 10 years of clinical experience. Artif Organs 1996; 20: 303-310.

[14] Pfefferkorn TK, Knuppel HP, Jaeger BR, et al. Increased cerebral $\mathrm{CO}(2)$ reactivity after heparin-mediated extracorporal LDL precipitation (HELP) in patients with coronary heart disease and hyperlipidemia. Stroke 1999; 30: 1802-1806.

[15] Mellwig KP, van BF, Schmidt HK, et al. Improved coronary vasodilatatory capacity by H.E.L.P. apheresis: comparing initial and chronic treatment. Ther Apher Dial 2006; 10: 510-517.

[16] Cunningham DR, Goetzinger CP. Extra-high frequency hearing loss and hyperlipidemia. Audiology 1974; 13: 470-484.

[17] Morizono T, Sikora MA, Ward WD, et al. Hyperlipidemia and noise in the chinchilla. Acta Otolaryngol 1985; 99: 516-524.

[18] Saito T, Sato K, Saito H. An experimental study of auditory dysfunction associated with hyperlipoproteinemia. Arch Otorhinolaryngol 1986; 243: 242-245.

[19] Gratton MA, Wright CG. Alterations of inner ear morphology in experimental hypercholesterolemia. Hear Res 1992; 61: 97-105.

[20] Fessenden JD, Schacht J. The nitric oxide/cyclic GMP pathway: a potential major regulator of cochlear physiology. Hear Res 1998; 118: 168-176.

[21] Shiraishi T, Kubo T, Okumura S, et al. Hearing recovery in sudden deafness patients using a modified defibrinogenation therapy. Acta Otolaryngol Suppl 1993; 501: 46-50. 